TRANSACTIONS OF THE

AMERICAN MATHEMATICAL SOCIETY

Volume 362, Number 1, January 2010, Pages 1-18

S 0002-9947(09)04691-1

Article electronically published on July 31, 2009

\title{
ON THE HECKE ALGEBRAS AND THE COLORED HOMFLY POLYNOMIAL
}

\author{
XIAO-SONG LIN AND HAO ZHENG
}

\begin{abstract}
The colored HOMFLY polynomial is the quantum invariant of oriented links in $S^{3}$ associated with irreducible representations of the quantum group $U_{q}\left(\mathrm{sl}_{N}\right)$. In this paper, using an approach to calculate quantum invariants of links via the cabling-projection rule, we derive a formula for the colored HOMFLY polynomial in terms of the characters of the Hecke algebras and Schur polynomials. The technique leads to a fairly simple formula for the colored HOMFLY polynomial of torus links. This formula allows us to test the Labastida-Mariño-Vafa conjecture, which reveals a deep relationship between Chern-Simons gauge theory and string theory, on torus links.
\end{abstract}

\section{INTRODUCTION}

In the abstract of his seminal paper [7, V. Jones wrote: "By studying representations of the braid group satisfying a certain quadratic relation we obtain a polynomial invariant in two variables for oriented links. ... The two-variable polynomial was first discovered by Freyd-Yetter, Lickorish-Millet, Ocneanu, Hoste, and Przytycki-Traczyk." This two variable link polynomial $P_{\mathcal{L}}(t, \nu)$, commonly referred to as the HOMFLY polynomial for an oriented link $\mathcal{L}$ in $S^{3}$, is characterized by the following crossing changing formula:

$$
\begin{aligned}
& P_{\text {unknot }}(t, \nu)=1, \\
& \nu^{-1 / 2} P_{\mathcal{L}_{+}}(t, \nu)-\nu^{1 / 2} P_{\mathcal{L}_{-}}(t, \nu)=\left(t^{-1 / 2}-t^{1 / 2}\right) P_{\mathcal{L}_{0}}(t, \nu) .
\end{aligned}
$$

Since then, this two variable link polynomial has been generalized to the quantum invariant associated with irreducible representations of the quantum group $U_{q}\left(\mathrm{sl}_{N}\right)$, with the variables $t^{1 / 2}=q^{-1}$ and $\nu^{1 / 2}=q^{-N}$. We will refer to this generalization as the colored HOMFLY polynomial.

Despite the fact that the theory of quantum invariants of links is by now well developed, the computation of the colored HOMFLY polynomial is still extremely challenging. Besides the trivial links, a general formula seems to exist in the mathematics literature only for the Hopf link [14. In the physics literature, Witten's Chern-Simons path integral with the gauge group $\mathrm{SU}_{N}$ 21] offers an intrinsic but not rigorous definition of the colored HOMFLY polynomial. There is a conjectured relationship between the $1 / N$ expansion of Chern-Simons theory and the Gromov-Witten invariants of certain noncompact Calabi-Yau 3-folds. See [5], 16]

Received by the editors August 4, 2006.

2000 Mathematics Subject Classification. Primary 57M27, $20 \mathrm{C} 08$.

The first author was supported in part by NSF grants DMS-0404511.

The second author was supported in part by an NSFC grant.

(C)2009 American Mathematical Society 
for example. Motivated by this conjectured relationship, Labastida, Mariño and Vafa proposed a precise conjecture about the structure of their reformulation of the colored HOMFLY polynomial [1], 12. See Section 5. A formula for the colored HOMFLY polynomial for torus knots is given in [10, which was used to test the Labastida-Mariño-Vafa conjecture on torus knots.

In this paper, using an approach to calculate quantum invariants of links via the cabling-projection rule, we derive a formula for the colored HOMFLY polynomial in terms of the characters of the Hecke algebras and Schur polynomials. See Theorem 4.3. An important feature of this formula is that the character of the Hecke algebra is free of the variable $\nu$ and the Schur polynomial is independent of the link $\mathcal{L}$. We think that this separation of the variable $\nu$ and the link $\mathcal{L}$ might be important for a possible proof of the Labastida-Mariño-Vafa conjecture.

Our technique leads to a fairly simple formula for the colored HOMFLY polynomial of torus links. See Theorem 5.1. Using our formula, the Labastida-Mariño-Vafa conjecture can be tested on several infinite families of torus links. Our calculation also suggests a new structure of the reformulated colored HOMFLY polynomial of torus links: it is equivalent to a family of polynomials in $\mathbb{Z}\left[t^{ \pm 1}\right]$ invariant under the transformation $t \rightarrow t^{-1}$. See Conjecture 6.2 and the examples following it.

\section{LINK INVARIANTS FROM QUANTUM GROUPS}

In this section, we give a brief review of the quantum group invariants of links. See [8], [17, 19] for details.

Let $\mathfrak{g}$ be a complex simple Lie algebra and let $q$ be a nonzero complex number which is not a root of unity. Let $U_{q}(\mathfrak{g})$ denote the quantum enveloping algebra of $\mathfrak{g}$. The ribbon category structure of the set of finite dimensional complex representations of $U_{q}(\mathfrak{g})$ provides the following objects.

1. Associated to each pair of $U_{q}(\mathfrak{g})$-modules $V, W$ is a natural isomorphism (the braiding) $\check{R}_{V, W}: V \otimes W \rightarrow W \otimes V$ such that

$$
\begin{aligned}
& \check{R}_{U \otimes V, W}=\left(\check{R}_{U, W} \otimes \operatorname{id}_{V}\right)\left(\operatorname{id}_{U} \otimes \check{R}_{V, W}\right), \\
& \check{R}_{U, V \otimes W}=\left(\operatorname{id}_{V} \otimes \check{R}_{U, W}\right)\left(\check{R}_{U, V} \otimes \operatorname{id}_{W}\right)
\end{aligned}
$$

hold for all $U_{q}(\mathfrak{g})$-modules $U, V, W$. The naturality means

$$
(y \otimes x) \check{R}_{V, W}=\check{R}_{V^{\prime}, W^{\prime}}(x \otimes y)
$$

for $x \in \operatorname{Hom}_{U_{q}(\mathfrak{g})}\left(V, V^{\prime}\right), y \in \operatorname{Hom}_{U_{q}(\mathfrak{g})}\left(W, W^{\prime}\right)$. These equalities imply the braiding relation

$$
\begin{aligned}
\left(\check{R}_{V, W} \otimes \operatorname{id}_{U}\right)\left(\operatorname{id}_{V} \otimes \check{R}_{U, W}\right)\left(\check{R}_{U, V} \otimes \operatorname{id}_{W}\right) \\
=\left(\operatorname{id}_{W} \otimes \check{R}_{U, V}\right)\left(\check{R}_{U, W} \otimes \operatorname{id}_{V}\right)\left(\operatorname{id}_{U} \otimes \check{R}_{V, W}\right) .
\end{aligned}
$$

2. There exists an element $K_{2 \rho} \in U_{q}(\mathfrak{g})$ (the enhancement of $\check{R}$, here $\rho$ means the half-sum of all positive roots of $\mathfrak{g}$ ) such that

$$
K_{2 \rho}(v \otimes w)=K_{2 \rho}(v) \otimes K_{2 \rho}(w)
$$

for $v \in V, w \in W$. Moreover, for every $z \in \operatorname{End}_{U_{q}(\mathfrak{g})}(V \otimes W)$ with $z=\sum_{i} x_{i} \otimes y_{i}$, $x_{i} \in \operatorname{End}(V), y_{i} \in \operatorname{End}(W)$ one has the (partial) quantum trace

$$
\operatorname{tr}_{W}(z)=\sum_{i} \operatorname{tr}\left(y_{i} K_{2 \rho}\right) \cdot x_{i} \in \operatorname{End}_{U_{q}(\mathfrak{g})}(V) .
$$


3. Associated to each $U_{q}(\mathfrak{g})$-module $V$ is a natural isomorphism (the ribbon structure) $\theta_{V}: V \rightarrow V$ satisfying

$$
\theta_{V}^{ \pm 1}=\operatorname{tr}_{V} \check{R}_{V, V}^{ \pm 1}
$$

The naturality means

$$
x \cdot \theta_{V}=\theta_{V^{\prime}} \cdot x
$$

for $x \in \operatorname{Hom}_{U_{q}(\mathfrak{g})}\left(V, V^{\prime}\right)$.

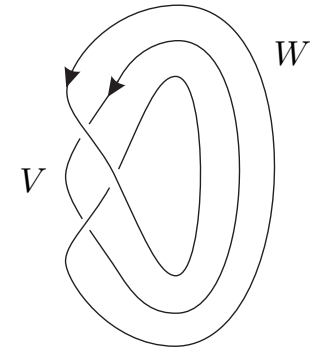

$\mathcal{L}$

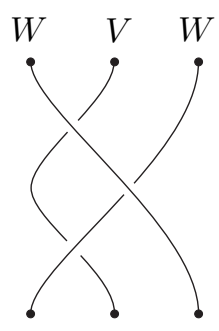

$\beta$

With these objects, one constructs the quantum group invariants of links as follows. Let $\mathcal{L}$ be an oriented link with the components $\mathcal{L}_{1}, \ldots, \mathcal{L}_{l}$ labeled by the $U_{q}(\mathfrak{g})$-modules $V_{1}, \ldots, V_{l}$, respectively. Choose a closed braid representative $\hat{\beta}$ of $\mathcal{L}$ with $\beta \in B_{n}$ being an $n$-strand braid. Assign to each positive (resp. negative) crossing of $\beta$ an isomorphism $\check{R}_{V, W}$ (resp. $\check{R}_{W, V}^{-1}$ ) where $V, W$ are the $U_{q}(\mathfrak{g}$ )-modules labeling the two outgoing strands of the crossing.
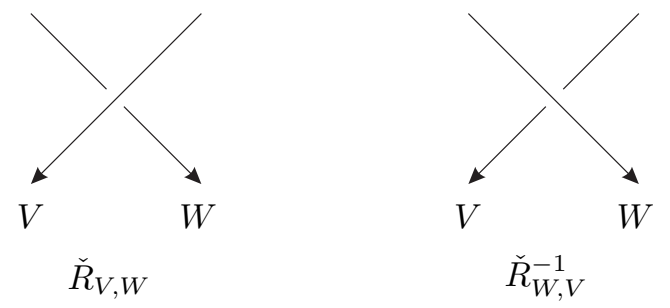

Then the braid $\beta$ gives rise to an isomorphism

$$
h_{V_{1}^{\prime}, \ldots, V_{n}^{\prime}}(\beta) \in \operatorname{End}_{U_{q}(\mathfrak{g})}\left(V_{1}^{\prime} \otimes \cdots \otimes V_{n}^{\prime}\right),
$$

where $V_{1}^{\prime}, \ldots, V_{n}^{\prime}$ are the $U_{q}(\mathfrak{g})$-modules labeling the strands of $\beta$, and the quantum trace

$$
\operatorname{tr}_{V_{1}^{\prime} \otimes \cdots \otimes V_{n}^{\prime}} h_{V_{1}^{\prime}, \ldots, V_{n}^{\prime}}(\beta)
$$

defines a framing dependent link invariant of $\mathcal{L}$.

Example 2.1. The link shown in the above figure has two components, labeled by $W$ and $V$ respectively. It is the closure of $\beta=\sigma_{1}^{-1} \sigma_{2}^{-1} \sigma_{1} \in B_{3}$, which gives rise to an isomorphism

$$
h_{W, V, W}(\beta)=\left(\check{R}_{W, V}^{-1} \otimes \operatorname{id}_{W}\right)\left(\operatorname{id}_{V} \otimes \check{R}_{W, W}^{-1}\right)\left(\check{R}_{W, V} \otimes \operatorname{id}_{W}\right) .
$$

Thus the link invariant is

$$
\operatorname{tr}_{W \otimes V \otimes W}\left(\check{R}_{W, V}^{-1} \otimes \operatorname{id}_{W}\right)\left(\operatorname{id}_{V} \otimes \check{R}_{W, W}^{-1}\right)\left(\check{R}_{W, V} \otimes \operatorname{id}_{W}\right) .
$$


To eliminate the framing dependency, one should require the modules $V_{1}, \ldots, V_{l}$ to be irreducible; hence the isomorphisms $\theta_{V_{1}}, \ldots, \theta_{V_{l}}$ are multiples of the identity and may be regarded as scalars. Let $w\left(\mathcal{L}_{i}\right)$ be the writhe of $\mathcal{L}_{i}$ in $\beta$, i.e. the number of positive crossings minus the number of negative crossings. Then the quantity

$$
I_{\mathcal{L} ; V_{1}, \ldots, V_{l}}=\theta_{V_{1}}^{-w\left(\mathcal{L}_{1}\right)} \cdots \theta_{V_{l}}^{-w\left(\mathcal{L}_{l}\right)} \operatorname{tr}_{V_{1}^{\prime} \otimes \cdots \otimes V_{n}^{\prime}} h_{V_{1}^{\prime}, \ldots, V_{n}^{\prime}}(\beta)
$$

defines a framing independent link invariant.

When the link involved is the unknot, it is easy to see that

$$
I_{\text {unknot } ; V}=\operatorname{tr}_{V} \operatorname{id}_{V} \text {. }
$$

This quantity is regarded as the quantum version of the classical dimension of $V$, referred to as the quantum dimension of $V$ and denoted by $\operatorname{dim}_{q} V$.

\section{Centralizer algebra And CABling-Projection Rule}

In general, the isomorphism $\check{R}_{V, W}$ is very complicated when the dimensions of $V, W$ are larger, so it is not practical to compute the link invariants from their definition. However, on the other hand, general representations of a simple Lie algebra $\mathfrak{g}$ (thus its quantum deformation $U_{q}(\mathfrak{g})$ ) are often realized as components of tensor products of some simple ones. For example, irreducible representations of $U_{q}\left(\mathrm{sl}_{N}\right)$ are always the components of some tensor products of the fundamental representation.

In this section, we follow this observation and develop a cabling-projection rule to break down the complexity of general $\check{R}$. For this purpose we need the notion of centralizer algebra.

The centralizer algebras of the modules of simple Lie algebras have played an important role in representation theory. Parts of their quantum version were studied in [2, [13], 20]. In the case of $U_{q}\left(\mathrm{sl}_{N}\right)$, the situation is desirable. The centralizer algebras are nothing but the subalgebras of the Hecke algebras of type $A$.

Let $V$ be a $U_{q}(\mathfrak{g})$-module. The centralizer algebra of $V^{\otimes n}$ is defined as

$$
\mathcal{C}_{n}(V)=\operatorname{End}_{U_{q}(\mathfrak{g})}\left(V^{\otimes n}\right)=\left\{x \in \operatorname{End}\left(V^{\otimes n}\right) \mid x y=y x, \forall y \in U_{q}(\mathfrak{g})\right\} .
$$

It is immediate from the definition that $\mathcal{C}_{n}(V)$ is a finite dimensional von Neumann algebra; i.e. the algebra is isomorphic to a direct sum of matrix algebras. Indeed, if $V^{\otimes n}$ admits the irreducible decomposition

$$
V^{\otimes n}=\bigoplus_{\lambda \in \Lambda} d_{\lambda} \cdot V_{\lambda}
$$

by Schur's lemma we have

$$
\mathcal{C}_{n}(V)=\bigoplus_{\lambda \in \Lambda} \mathcal{C}_{\lambda}
$$

where $\mathcal{C}_{\lambda}=\operatorname{End}_{U_{q}(\mathfrak{g})}\left(d_{\lambda} V_{\lambda}\right)$ is a full $d_{\lambda} \times d_{\lambda}$ matrix algebra. Since each matrix algebra admits a unique irreducible representation, via the above decomposition the irreducible representations of $\mathcal{C}_{n}(V)$ are naturally indexed by $\Lambda$.

Let $\zeta^{\lambda}$ denote the character of the irreducible representation of $\mathcal{C}_{n}(V)$ indexed by $\lambda \in \Lambda$.

Lemma 3.1. For every $x \in \mathcal{C}_{n}(V)$ we have

$$
\operatorname{tr}_{V \otimes n} x=\sum_{\lambda \in \Lambda} \zeta^{\lambda}(x) \cdot \operatorname{dim}_{q} V_{\lambda} .
$$


Proof. Let $\pi_{\lambda}$ be the unit of $\mathcal{C}_{\lambda}$. Then $\pi_{\lambda} x$ is a matrix in $\mathcal{C}_{\lambda}$, whose usual trace $\operatorname{tr} \pi_{\lambda} x$ is precisely $\zeta^{\lambda}(x)$. Therefore,

$$
\operatorname{tr}_{V^{\otimes n}} x=\sum_{\lambda \in \Lambda} \operatorname{tr} \pi_{\lambda} x \cdot \operatorname{tr}_{V_{\lambda}} \operatorname{id}_{V_{\lambda}}=\sum_{\lambda \in \Lambda} \zeta^{\lambda}(x) \cdot \operatorname{dim}_{q} V_{\lambda} .
$$

A projection (or idempotent) of $\mathcal{C}_{n}(V)$ is an element $p \in \mathcal{C}_{n}(V)$ satisfying the idempotent equation $p^{2}=p$. By definition, an element $p \in \mathcal{C}_{n}(V)$ is a projection if and only if it is, restricted on each $\mathcal{C}_{\lambda}$, diagonalizable and has the only possible eigenvalues 0 and 1 . It is clear that for each projection $p \in \mathcal{C}_{n}(V)$,

$$
p V^{\otimes n} \cong \bigoplus_{\lambda \in \Lambda} \zeta^{\lambda}(p) \cdot V_{\lambda} .
$$

A projection $p \in \mathcal{C}_{n}(V)$ is called minimal (or primitive) if $p V^{\otimes n} \cong V_{\lambda}$ for some $\lambda \in \Lambda$.

Let $h_{V}$ be the homomorphism

$$
h_{V}: \mathbb{C} B_{n} \rightarrow \mathcal{C}_{n}(V), \quad \sigma_{i} \mapsto \mathrm{id}_{V^{\otimes(i-1)}} \otimes R_{V, V} \otimes \mathrm{id}_{V^{\otimes(n-i-1)}} .
$$

The following lemma makes it possible to recover general $\check{R}, \theta$ from specific ones.

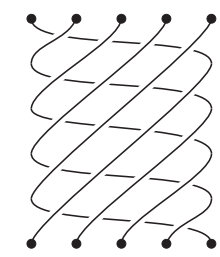

$\Delta_{5}^{2}$

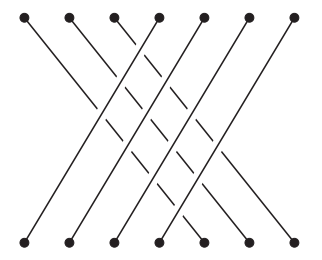

$\chi_{3,4}$

Lemma 3.2. Let $\Delta_{n}^{2}=\left(\sigma_{1} \sigma_{2} \cdots \sigma_{n-1}\right)^{n} \in B_{n}$ and $\chi_{n, n^{\prime}}=\prod_{i=1}^{n^{\prime}}\left(\sigma_{i+n-1} \sigma_{i+n-2} \cdots \sigma_{i}\right)$ $\in B_{n+n^{\prime}}$ denote the full twist braid and the $\left(n, n^{\prime}\right)$-crossing braid, respectively. Let $p \in \mathcal{C}_{n}(V), p^{\prime} \in \mathcal{C}_{n^{\prime}}(V)$ be projections and $U=p V^{\otimes n}, W=p^{\prime} V^{\otimes n^{\prime}}$. We have

$$
\begin{aligned}
& h_{V}\left(\chi_{n, n^{\prime}}\right) \cdot\left(p \otimes p^{\prime}\right)=\check{R}_{W, U} \oplus 0_{\operatorname{Ker} p \otimes p^{\prime}}, \\
& \left(\theta_{V}\right)^{\otimes n} \cdot h_{V}\left(\Delta_{n}^{2}\right) \cdot p=\theta_{U} \oplus 0_{\text {Ker } p} .
\end{aligned}
$$

Proof. Applying the identities (2.1), (2.3) and (2.6) inductively, we have

$$
\begin{aligned}
& \check{R}_{V \otimes n^{\prime}, V \otimes n}=h_{V}\left(\chi_{n, n^{\prime}}\right), \\
& \theta_{V^{\otimes n}}=\operatorname{tr}_{V}^{\otimes n} \check{R}_{V^{\otimes n}, V \otimes n}=\left(\theta_{V}\right)^{\otimes n} \cdot h_{V}\left(\Delta_{n}^{2}\right) .
\end{aligned}
$$

Then from the naturality of $\check{R}$ and $\theta$ the lemma follows.

As an easy consequence of (3.8) and the naturality of $\check{R}$, we have the cablingprojection rule.

Lemma 3.3. Let $\beta \in B_{m}$ be a braid and $p_{i} \in \mathcal{C}_{n_{i}}(V), i=1, \ldots, m$ be projections such that $p_{i}=p_{j}$ whenever the $i$-th strand of $\beta$ ends at the $j$-th point. Moreover, let $V_{i}=p_{i} V^{\otimes n_{i}}, n=n_{1}+\cdots+n_{m}$ and $\beta^{\left(n_{1}, \ldots, n_{m}\right)} \in B_{n}$ be the braid obtained by cabling the $i$-th strand of $\beta$ to $n_{i}$ parallel ones. Then

$$
h_{V}\left(\beta^{\left(n_{1}, \ldots, n_{m}\right)}\right) \cdot\left(p_{1} \otimes \cdots \otimes p_{m}\right)=h_{V_{1}, \ldots, V_{m}}(\beta) \oplus 0_{\operatorname{Ker} p_{1} \otimes \cdots \otimes p_{m}} ;
$$


thus

$$
\operatorname{tr}_{V_{1} \otimes \cdots \otimes V_{m}} h_{V_{1}, \ldots, V_{m}}(\beta)=\operatorname{tr}_{V \otimes n} h_{V}\left(\beta^{\left(n_{1}, \ldots, n_{m}\right)}\right) \cdot\left(p_{1} \otimes \cdots \otimes p_{m}\right) .
$$

With the above lemmas, one is able to re-express the link invariant (2.12), by choosing a suitable $U_{q}(\mathfrak{g})$-module $V$, in terms of much more accessible objects: the characters and projections of the centralizer algebras $\mathcal{C}_{n}(V)$ and the quantum traces of $U_{q}(\mathfrak{g})$-modules. In the next section, we present a detailed realization of this approach for the case $\mathfrak{g}=\mathrm{sl}_{N}$.

\section{Hecke Algebras and COlOREd HOMFLy polynomial}

In the rest part of this paper we will extensively apply the facts concerning the Hecke algebras [3, [15, the quantum enveloping algebras $U_{q}\left(\mathrm{sl}_{N}\right) \underline{9}$ and symmetric functions [18].

The Hecke algebra $\mathcal{H}_{n}(q)$ of type $A_{n-1}$ is the complex algebra with generators $g_{1}, g_{2}, \ldots, g_{n-1}$ and relations

$$
\begin{array}{ll}
g_{i} g_{j}=g_{j} g_{i}, & |i-j| \geq 2, \\
g_{i} g_{j} g_{i}=g_{j} g_{i} g_{j}, & |i-j|=1, \\
\left(g_{i}-q\right)\left(g_{i}+q^{-1}\right)=0, & i=1,2, \ldots, n-1 .
\end{array}
$$

Note that, when $q=1$, the Hecke algebra $\mathcal{H}_{n}(q)$ is nothing new but the group algebra $\mathbb{C} \Sigma_{n}$ of the symmetric group. In fact, if $q$ is nonzero and not a root of unity, then we still have the isomorphism $\mathcal{H}_{n}(q) \cong \mathbb{C} \Sigma_{n}$ and $\mathcal{H}_{n}(q)$ also canonically decomposes as

$$
\mathcal{H}_{n}(q)=\bigoplus_{\lambda \vdash n} \mathcal{H}_{\lambda}(q)
$$

with each $\mathcal{H}_{\lambda}(q)$ being a matrix algebra.

Here we fix some notation of combinatorics. A composition $\mu$ of $n$, denoted by $\mu \models n$, is a sequence of nonnegative integers $\left(\mu_{1}, \mu_{2}, \ldots\right)$ such that $\sum_{i} \mu_{i}=n$. The length $\ell(\mu)$ of $\mu$ is the maximal index $i$ with $\mu_{i}$ nonzero. If, in addition, $\mu_{1} \geq \mu_{2} \geq \cdots$, then $\mu$ is also called a partition and one writes $\mu \vdash n$ and $|\mu|=n$.

It is a standard result that the centralizer algebras of $\mathrm{sl}_{N}$-modules are canonically subalgebras of $\mathbb{C} \Sigma_{n}$. So it is not surprising to see that the centralizer algebras of $U_{q}\left(\mathrm{sl}_{N}\right)$-modules are realized as subalgebras of $\mathcal{H}_{n}(q)$, the quantum deformation of $\mathbb{C} \Sigma_{n}$.

Now fix $\mathfrak{g}=\operatorname{sl}_{N}$ and let $V$ be the module of the fundamental representation of $U_{q}\left(\mathrm{sl}_{N}\right)$. With a suitable basis $\left\{v_{1}, \ldots, v_{N}\right\}$ of $V$ and generators $\left\{K_{i}^{ \pm 1}, E_{i}, F_{i} \mid 1 \leq\right.$ $i \leq N-1\}$ of $U_{q}\left(\mathrm{sl}_{N}\right)$, the fundamental representation is given by the matrices

$$
\begin{aligned}
K_{i} & \mapsto q E_{i i}+q^{-1} E_{i+1, i+1}+\sum_{j \neq i} E_{j j}, \\
E_{i} & \mapsto E_{i, i+1}, \\
F_{i} & \mapsto E_{i+1, i},
\end{aligned}
$$

where $E_{i j}$ is the $N \times N$ matrix with 1 in the $(i, j)$-position and 0 elsewhere. We also have

$$
\begin{aligned}
& q^{1 / N} \theta_{V}=q^{N} \cdot \operatorname{id}_{V}, \\
& K_{2 \rho}\left(v_{i}\right)=q^{N+1-2 i} v_{i},
\end{aligned}
$$


and

$$
q^{1 / N} \check{R}_{V, V}\left(v_{i} \otimes v_{j}\right)= \begin{cases}q v_{i} \otimes v_{j}, & i=j, \\ v_{j} \otimes v_{i}, & i<j, \\ v_{j} \otimes v_{i}+\left(q-q^{-1}\right) v_{i} \otimes v_{j}, & i>j .\end{cases}
$$

It is straightforward to verify that the homomorphism $h_{V}: \mathbb{C} B_{n} \rightarrow \mathcal{C}_{n}(V)$ factors through $\mathcal{H}_{n}(q)$ via

$$
q^{1 / N} \sigma_{i} \mapsto g_{i} \mapsto q^{1 / N} h_{V}\left(\sigma_{i}\right)
$$

Therefore, $V^{\otimes n}$ is a module of both $U_{q}\left(\operatorname{sl}_{N}\right)$ and $\mathcal{H}_{n}(q)$, and the two algebras act commutatively on $V^{\otimes n}$. For convenience, we introduce an $N$-independent homomorphism

$$
h: \mathbb{C} B_{n} \rightarrow \mathcal{H}_{n}(q), \quad \sigma_{i} \mapsto g_{i} .
$$

Let $S^{\lambda}$ denote the irreducible module of $\mathcal{H}_{n}(q)$ indexed by the partition $\lambda \vdash n$ and let $\zeta^{\lambda}$ denote its character. Fix a minimal projection $p_{\lambda} \in \mathcal{H}_{\lambda}(q)$ for each $\lambda \vdash n$. Let $V_{\lambda}$ denote the irreducible $U_{q}\left(\mathrm{sl}_{N}\right)$-module, whose highest weight vector $v$ behaves like $K_{i}(v)=q^{\lambda_{i}-\lambda_{i+1}} v$ if $\ell(\lambda) \leq N$ and is 0 otherwise.

We state below two important facts concerning the $U_{q}\left(\mathrm{sl}_{N}\right)$-module $V^{\otimes n}$. One is the irreducible decomposition of the $U_{q}\left(\mathrm{sl}_{N}\right)$-module,

$$
V^{\otimes n}=\bigoplus_{\lambda \vdash n, \ell(\lambda) \leq N} \operatorname{dim} S^{\lambda} \cdot V_{\lambda},
$$

in which the subspace $\operatorname{dim} S^{\lambda} \cdot V_{\lambda}$ is $\mathcal{H}_{n}(q)$-invariant and, as an $\mathcal{H}_{n}(q)$-module, consists of only $S^{\lambda}$-components. Notice that the $U_{q}\left(\mathrm{sl}_{N}\right)$-modules $\left\{V_{\lambda} \mid \lambda \vdash n, \ell(\lambda) \leq\right.$ $N\}$ are mutually inequivalent. Comparing (4.9), (4.2) with (3.2), (3.3), we have immediately

$$
\mathcal{C}_{n}(V)=\bigoplus_{\lambda \vdash n, \ell(\lambda) \leq N} \mathcal{H}_{\lambda}(q) .
$$

Moreover, for every partition $\lambda \vdash n$,

$$
p_{\lambda} V^{\otimes n} \cong V_{\lambda} .
$$

The other fact is the weight decomposition of the $U_{q}\left(\mathrm{sl}_{N}\right)$-module,

$$
V^{\otimes n}=\bigoplus_{\mu \models n, \ell(\mu) \leq N} M^{\mu},
$$

where

$$
M^{\mu}=\left\{v \in V^{\otimes n} \mid K_{i}(v)=q^{\mu_{i}-\mu_{i+1}} v\right\} .
$$

Moreover, the dimensions of the weight spaces of $V_{\lambda}$ for $\lambda \vdash n$,

$$
K_{\lambda \mu}=\operatorname{dim}\left(p_{\lambda} V^{\otimes n} \cap M^{\mu}\right),
$$

are encoded in the Schur polynomial as

$$
s_{\lambda}\left(z_{1}, \ldots, z_{N}\right)=\sum_{\mu \models n, \ell(\mu) \leq N} K_{\lambda \mu} \cdot \prod_{j=1}^{N} z_{j}^{\mu_{j}} .
$$

Indeed, $M^{\mu}$ is nothing but the subspace of $V^{\otimes n}$ spanned by the vectors $v_{i_{1}} \otimes \cdots \otimes v_{i_{n}}$ in which $v_{i}$ appears precisely $\mu_{i}$ times. It is clear that $M^{\mu}$ is $\mathcal{H}_{n}(q)$-invariant. In the 
literature, $M^{\mu}$ is called the permutation module and the integers $K_{\lambda \mu}$ are referred to as Kostka numbers.

Minimal projections of the Hecke algebras were extensively studied in the literature. See, for instance, [1], 4], [6], [15]. For the reader's convenience, we present a possible choice of them as follows (see [1, Theorem 4.7]).

Regard partitions as Young diagrams. For a partition $\lambda \vdash n$, let $\lambda^{\vee}$ denote the transpose of $\lambda$ and let $w_{\lambda} \in \Sigma_{n}$ be the permutation which sends the symbol in the cell $(i, j)$ of $\lambda$ to the symbol in the cell $(j, i)$ of $\lambda^{\vee}$ (with symbols assigned as in the figure below).

$$
\begin{array}{|l|l|l|l|}
\hline 1 & 2 & 3 & 4 \\
\cline { 1 - 2 } 5 & 6 & 7 & \multicolumn{1}{|c}{} \\
\cline { 1 - 2 } 8 & 9 & \multicolumn{2}{|c}{} \\
\cline { 1 - 2 } & &
\end{array}
$$$$
\lambda=(4,3,2)
$$

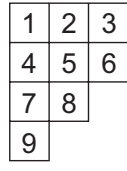

$\lambda^{\vee}=(3,3,2,1)$

Recall that for each permutation $w \in \Sigma_{n}$, there is a unique element $g_{w} \in \mathcal{H}_{n}(q)$ such that $g_{w}=g_{i_{1}} g_{i_{2}} \cdots g_{i_{k}}$ for a reduced word $w=s_{i_{1}} s_{i_{2}} \cdots s_{i_{k}}$ (where $s_{j}=(j, j+1)$ ). Define

$$
\begin{array}{ll}
E_{n}=\sum_{w \in \Sigma_{n}} q^{\ell(w)} g_{w}, & E_{\lambda}=E_{\lambda_{1}} \otimes E_{\lambda_{2}} \otimes \cdots \otimes E_{\lambda_{\ell(\lambda)}}, \\
F_{n}=\sum_{w \in \Sigma_{n}}\left(-q^{-1}\right)^{\ell(w)} g_{w}, & F_{\lambda}=F_{\lambda_{1}} \otimes F_{\lambda_{2}} \otimes \cdots \otimes F_{\lambda_{\ell(\lambda)}}, \\
{[n]_{q}=\frac{q^{n}-q^{-n}}{q-q^{-1}},} & \alpha_{\lambda}=\prod_{(i, j) \in \lambda} q^{j-i}\left[\lambda_{i}+\lambda_{j}^{\vee}-i-j+1\right]_{q} .
\end{array}
$$

In the above notation, a possible choice of $p_{\lambda}$ is

$$
p_{\lambda}=\frac{E_{\lambda} \cdot g_{w_{\lambda}} \cdot F_{\lambda^{\vee}} \cdot g_{w_{\lambda}}^{-1}}{\alpha_{\lambda}} .
$$

For example,

$$
\begin{aligned}
& p_{(2)}=\frac{1+q g_{1}}{1+q^{2}}, \quad p_{(1,1)}=\frac{1-q^{-1} g_{1}}{q^{-2}+1}, \\
& p_{(3)}=\frac{1+q g_{1}+q g_{2}+q^{2} g_{1} g_{2}+q^{2} g_{2} g_{1}+q^{3} g_{1} g_{2} g_{1}}{1+2 q^{2}+2 q^{4}+q^{6}}, \\
& p_{(2,1)}=\frac{\left(1+q g_{1}\right) g_{2}\left(1-q^{-1} g_{1}\right) g_{2}^{-1}}{q^{-2}+1+q^{2}}, \\
& p_{(1,1,1)}=\frac{1-q^{-1} g_{1}-q^{-1} g_{2}+q^{-2} g_{1} g_{2}+q^{-2} g_{2} g_{1}-q^{-3} g_{1} g_{2} g_{1}}{q^{-6}+2 q^{-4}+2 q^{-2}+1} .
\end{aligned}
$$

The following theorem is important for later computations.

Theorem 4.1 (Aiston-Morton [1, Theorem 5.5]). For each partition $\lambda \vdash n$ with $\ell(\lambda) \leq N$, one has

$$
\theta_{V_{\lambda}}=q^{\kappa_{\lambda}+n N-n^{2} / N} \cdot \operatorname{id}_{V_{\lambda}},
$$

where

$$
\kappa_{\lambda}=\sum_{i=1}^{\ell(\lambda)} \sum_{j=1}^{\lambda_{i}} 2(j-i) .
$$


The next proposition is a strong version of Lemma 3.1. Equation (4.20) holds even for $x \notin \mathcal{C}_{n}(V)$.

Proposition 4.2. We have

$$
\operatorname{dim}_{q} V_{\lambda}=s_{\lambda}\left(q^{N-1}, q^{N-3}, \ldots, q^{-(N-1)}\right)
$$

thus for every $x \in \mathcal{H}_{n}(q)$,

$$
\operatorname{tr}_{V \otimes n} x=\sum_{\lambda \vdash n} \zeta^{\lambda}(x) \cdot s_{\lambda}\left(q^{N-1}, q^{N-3}, \ldots, q^{-(N-1)}\right) .
$$

Proof. By (2.4) and (4.5),$K_{2 \rho}$ acts as a scalar $\prod_{i=1}^{N} q^{(N+1-2 i) \mu_{j}}$ on $M^{\mu}$. Therefore, it follows from identity (4.15) that for each $\lambda \vdash n$,

$$
\operatorname{dim}_{q} V_{\lambda}=\sum_{\mu \models n, \ell(\mu) \leq N} K_{\lambda \mu} \cdot \prod_{i=1}^{N} q^{(N+1-2 i) \mu_{j}}=s_{\lambda}\left(q^{N-1}, q^{N-3}, \ldots, q^{-(N-1)}\right) .
$$

Now it is time to state our main result.

Theorem 4.3. Let $\mathcal{L}$ be an oriented link with l components $\mathcal{L}_{1}, \ldots, \mathcal{L}_{l}$. Suppose $\mathcal{L}$ is the closure of $\beta \in B_{m}$ and the $m$ strands of $\beta$ are living on $\mathcal{L}_{i_{1}}, \ldots, \mathcal{L}_{i_{m}}$, respectively. Then for partitions $\lambda^{i} \vdash n_{i}, i=1, \ldots, l$, we have

$$
\begin{gathered}
I_{\mathcal{L} ; V_{\lambda}, \ldots, V_{\lambda l}}=q^{-\sum_{i=1}^{l}\left(\kappa_{\lambda^{i}}+n_{i} N-n_{i}^{2} / N\right) w\left(\mathcal{L}_{i}\right)-w\left(\beta^{\left(n_{i_{1}}, \ldots, n_{i_{m}}\right)}\right) / N} \\
\cdot \sum_{\lambda \vdash n} \zeta^{\lambda}(x) \cdot s_{\lambda}\left(q^{N-1}, q^{N-3}, \ldots, q^{-(N-1)}\right),
\end{gathered}
$$

where $n=n_{i_{1}}+\cdots+n_{i_{m}}, \beta^{\left(n_{i_{1}}, \ldots, n_{i_{m}}\right)} \in B_{n}$ is the braid obtained by cabling the $j$-th strand of $\beta$ to $n_{i_{j}}$ parallel ones and $x=h\left(\beta^{\left(n_{i_{1}}, \ldots, n_{i_{m}}\right)}\right) \cdot\left(p_{\lambda^{i_{1}}} \otimes \cdots \otimes p_{\lambda^{i_{m}}}\right) \in \mathcal{H}_{n}(q)$.

Proof. Combine Lemma 3.3. Theorem 4.1 and Proposition 4.2 .

One notices that, on the right hand side of (4.21), there is an explicit factor $q^{1 / N}$ to the power

$$
\sum_{i=1}^{l} n_{i}^{2} w\left(\mathcal{L}_{i}\right)-w\left(\beta^{\left(n_{i_{1}}, \ldots, n_{i_{m}}\right)}\right)=-2 \sum_{i<j} n_{i} n_{j} \operatorname{lk}\left(\mathcal{L}_{i}, \mathcal{L}_{j}\right),
$$

where $\operatorname{lk}\left(\mathcal{L}_{i}, \mathcal{L}_{j}\right)$ are the linking numbers. As in [11, we drop this insignificant factor and regard the remaining part as a rational function of $q$ and $q^{N}$.

Definition 4.4. The colored HOMFLY polynomial $W_{\mathcal{L} ; \lambda^{1}, \ldots, \lambda^{l}}(t, \nu)$ with $\lambda^{i} \vdash n_{i}$ is a rational function of $t^{1 / 2}, \nu^{1 / 2}$ determined by

$$
\left.W_{\mathcal{L} ; \lambda^{1}, \ldots, \lambda^{l}}(t, \nu)\right|_{t^{1 / 2}=q^{-1}, \nu^{1 / 2}=q^{-N}}=q^{2 \sum_{i<j} n_{i} n_{j} \operatorname{lk}\left(\mathcal{L}_{i}, \mathcal{L}_{j}\right) / N} \cdot I_{\mathcal{L} ; V_{\lambda}, \ldots, V_{\lambda^{l}}} .
$$

Note that the definition means the components of the link $\mathcal{L}$ are labeled by partitions rather than $U_{q}\left(\mathrm{sl}_{N}\right)$-modules. When the labeling partitions are trivial (the unique partition of 1), the colored HOMFLY polynomial, up to a simple factor, specializes to the HOMFLY polynomial:

$$
P_{\mathcal{L}}(t, \nu)=\nu^{\operatorname{lk}(\mathcal{L})} \cdot \frac{t^{1 / 2}-t^{-1 / 2}}{\nu^{1 / 2}-\nu^{-1 / 2}} \cdot W_{\mathcal{L} ;(1), \ldots,(1)}(t, \nu) .
$$


Let $s_{\lambda}^{*}(t, \nu)$ be defined by (see (5.14) $)$

$$
\left.s_{\lambda}^{*}(t, \nu)\right|_{t^{1 / 2}=q^{-1}, \nu^{1 / 2}=q^{-N}}=s_{\lambda}\left(q^{N-1}, q^{N-3}, \ldots, q^{-(N-1)}\right) .
$$

Corollary 4.5. In the same notation as Theorem 4.3, we have

$$
W_{\mathcal{L} ; \lambda^{1}, \ldots, \lambda^{l}}(t, \nu)=\left.t^{\sum_{i=1}^{l} \kappa_{\lambda^{i}} w\left(\mathcal{L}_{i}\right) / 2} \cdot \nu^{\sum_{i=1}^{l} n_{i} w\left(\mathcal{L}_{i}\right) / 2} \cdot \sum_{\lambda \vdash n} \zeta^{\lambda}(x)\right|_{q=t^{-1 / 2}} \cdot s_{\lambda}^{*}(t, \nu) .
$$

\section{TORUS LINKS}

Let the notation be the same as in the previous section. In this section, we derive an explicit formula of the colored HOMFLY polynomial of torus links by applying Corollary 4.5 .

The torus link $T(r, k)$ is defined to be the closure of $\left(\delta_{r}\right)^{k}=\left(\sigma_{1} \cdots \sigma_{r-1}\right)^{k}$. They form the family of links that can be put on the standardly embedded torus $T \subset \mathbb{R}^{3}$. Some common links such as the trefoil knot $T(2,3)$ and the Hopf link $T(2,2)$ are included in this family.

Theorem 5.1. Let $\mathcal{L}$ be the torus link $T(r l, k l)$ with $r, k$ relatively prime. Let $\lambda^{i} \vdash n_{i}, i=1, \ldots, l$ be partitions and $n=n_{1}+\cdots+n_{l}$. Then

$$
W_{\mathcal{L} ; \lambda^{1}, \ldots, \lambda^{l}}(t, \nu)=t^{k r \sum_{i=1}^{l} \kappa_{\lambda^{i}} / 2} \cdot \nu^{k(r-1) n / 2} \cdot \sum_{\lambda \vdash r n} c_{\lambda^{1} \ldots \lambda^{l}}^{\lambda} \cdot t^{-k \kappa_{\lambda} / 2 r} \cdot s_{\lambda}^{*}(t, \nu),
$$

where $c_{\lambda^{1} \ldots \lambda^{l}}^{\lambda}$ are the integers determined by the equation

$$
\prod_{i=1}^{l} s_{\lambda^{i}}\left(x_{1}^{r}, x_{2}^{r}, \ldots\right)=\sum_{\lambda \vdash r n} c_{\lambda^{1} \ldots \lambda^{l}}^{\lambda} \cdot s_{\lambda}\left(x_{1}, x_{2}, \ldots\right) .
$$

The theorem is an easy consequence of following lemmas.

Lemma 5.2. For each partition $\lambda \vdash n$ we have

$$
h\left(\Delta_{n}^{2}\right) \cdot p_{\lambda}=q^{\kappa_{\lambda}} \cdot p_{\lambda} .
$$

Proof. Compare Lemma 3.2 with Theorem 4.1

Lemma 5.3. Let $\lambda^{i} \vdash n_{i}, i=1, \ldots, l$ be partitions and $n=n_{1}+\cdots+n_{l}$. Let $r, k$ be relatively prime integers and $\beta \in B_{r n}$ be the braid obtained by cabling the $(i l+j)$-th strand of $\left(\delta_{r l}\right)^{k l}$ to $n_{j}$ parallel ones. Then, for each partition $\lambda \vdash r n$ we have

$$
\zeta^{\lambda}\left(h(\beta) \cdot\left(p_{\lambda^{1}} \otimes \cdots \otimes p_{\lambda^{l}}\right)^{\otimes r}\right)=c_{\lambda^{1} \ldots \lambda^{l}}^{\lambda} \cdot q^{-k \sum_{i=1}^{l} \kappa_{\lambda^{i}}+k \kappa_{\lambda} / r} .
$$

Proof. Put $p=p_{\lambda^{1}} \otimes \cdots \otimes p_{\lambda^{l}}$ and let $\pi_{\lambda}$ be the unit of $\mathcal{H}_{\lambda}(q)$. Note that $\pi_{\lambda}$ is a central element of $\mathcal{H}_{r n}(q)$ and that $h(\beta)$ is commutative with $p^{\otimes r}$. We put

$$
x_{\lambda}=\pi_{\lambda} \cdot h(\beta) \cdot p^{\otimes r}
$$

regarded as a matrix in $\mathcal{H}_{\lambda}(q)$, its usual trace is

$$
\operatorname{tr} x_{\lambda}=\zeta^{\lambda}\left(h(\beta) \cdot p^{\otimes r}\right) .
$$

Notice that

$$
h\left(\beta^{r}\right)=h\left(\Delta_{r n}^{2 k}\right) \cdot\left(h\left(\Delta_{n_{1}}^{-2 k}\right) \otimes \cdots \otimes h\left(\Delta_{n_{l}}^{-2 k}\right)\right)^{\otimes r} .
$$


It follows from Lemma 5.2 that

$$
x_{\lambda}^{r}=\pi_{\lambda} \cdot h\left(\beta^{r}\right) \cdot p^{\otimes r}=q^{-k r \sum_{i=1}^{l} \kappa_{\lambda^{i}}+k \kappa_{\lambda}} \cdot \pi_{\lambda} \cdot p^{\otimes r} .
$$

Therefore, the eigenvalues of $x_{\lambda}$ are either 0 or $q^{-k \sum_{i=1}^{l} \kappa_{\lambda^{i}}+k \kappa_{\lambda} / r}$ times an $r$-th root of unity, for $\pi_{\lambda} \cdot p^{\otimes r} \in \mathcal{H}_{\lambda}(q)$ is also a projection. Since $\operatorname{tr} x_{\lambda}=\zeta^{\lambda}\left(h(\beta) \cdot p^{\otimes r}\right)$ is a rational function of $q$ in rational coefficients (clear from the choice (4.16) ), it follows that $\operatorname{tr} x_{\lambda}$ has to be $q^{-k \sum_{i=1}^{l} \kappa_{\lambda^{i}}+k \kappa_{\lambda} / r}$ times a rational number $a^{\lambda}$ which is independent of $q$.

Now, let $q \rightarrow 1$. Passing to the limit, $h(\beta)$ degenerates to a permutation $\tau \in \Sigma_{r n}$ which acts cyclicly on the $V^{\otimes n}$-factors of $V^{\otimes r n}=V^{\otimes n} \otimes \cdots \otimes V^{\otimes n}$. We have

$$
\begin{aligned}
& \sum_{\lambda \vdash r n} a^{\lambda} \cdot s_{\lambda}\left(z_{1}, \ldots, z_{N}\right) \\
& =\sum_{\lambda \vdash r n} a^{\lambda} \sum_{\mu \models r n, \ell(\mu) \leq N} \operatorname{dim}\left(p_{\lambda} V^{\otimes r n} \cap M^{\mu}\right) \cdot \prod_{j=1}^{N} z_{j}^{\mu_{j}} \quad \text { by (4.15) } \\
& =\sum_{\mu \models r n, \ell(\mu) \leq N} \sum_{\lambda \vdash r n} \zeta^{\lambda}\left(\tau \cdot p^{\otimes r}\right) \operatorname{dim}\left(p_{\lambda} V^{\otimes r n} \cap M^{\mu}\right) \cdot \prod_{j=1}^{N} z_{j}^{\mu_{j}} \quad \text { by (5.6) } \\
& =\left.\sum_{\mu \models r n, \ell(\mu) \leq N} \sum_{\lambda \vdash r n} \operatorname{tr}\left(\tau \cdot p^{\otimes r}\right)\right|_{\pi_{\lambda} V \otimes r n \cap M^{\mu}} \cdot \prod_{j=1}^{N} z_{j}^{\mu_{j}} \\
& =\left.\sum_{\mu \models r n, \ell(\mu) \leq N} \operatorname{tr} \tau\right|_{p^{\otimes r} V \otimes r n \cap M^{\mu}} \cdot \prod_{j=1}^{N} z_{j}^{\mu_{j}} \quad \text { by } \sum_{\lambda \vdash r n} \pi_{\lambda}=1 \\
& =\sum_{\mu \models n, \ell(\mu) \leq N} \operatorname{dim}\left(p V^{\otimes n} \cap M^{\mu}\right) \cdot \prod_{j=1}^{N} z_{j}^{r \mu_{j}} \\
& =\prod_{i=1}^{l}\left(\sum_{\mu \models n_{i}, \ell(\mu) \leq N} \operatorname{dim}\left(p_{\lambda^{i}} V^{\otimes n_{i}} \cap M^{\mu}\right) \cdot \prod_{j=1}^{N} z_{j}^{r \mu_{j}}\right) \\
& =\prod_{i=1}^{l} s_{\lambda^{i}}\left(z_{1}^{r}, \ldots, z_{N}^{r}\right) . \quad \text { by (4.15). }
\end{aligned}
$$

The third equality is by the fact that $\operatorname{dim}\left(p_{\lambda} V^{\otimes r n} \cap M^{\mu}\right)$ is the multiplicity of the $S^{\lambda}$-component in the $\mathcal{H}_{r n}(q)$-module $\pi_{\lambda} V^{\otimes r n} \cap M^{\mu}$ (see (4.9)).

Since the above equation holds for all $N$, we conclude that $a^{\lambda}=c_{\lambda^{1} \ldots \lambda^{l}}^{\lambda}$.

Remark 5.4. In the case $l=2$ and $r=1, k=0$, equation (5.4) specializes to

$$
\zeta^{\lambda}\left(p_{\lambda^{1}} \otimes p_{\lambda^{2}}\right)=c_{\lambda^{1} \lambda^{2}}^{\lambda}
$$

This implies that (see (3.6))

$$
V_{\lambda^{1}} \otimes V_{\lambda^{2}}=\bigoplus_{\lambda \vdash n} c_{\lambda^{1} \lambda^{2}}^{\lambda} \cdot V_{\lambda} .
$$

In the literature, the integers $c_{\lambda^{1} \lambda^{2}}^{\lambda}$ are referred to as Littlewood-Richardson coeffcients. 
Proof of Theorem 5.1. By Corollary 4.5 and Lemma 5.3, we have

$$
\begin{aligned}
& W_{\mathcal{L} ; \lambda^{1}, \ldots, \lambda^{l}}(t, \nu) \\
& =t^{\sum_{i=1}^{l} \kappa_{\lambda^{i}} k(r-1) / 2} \cdot \nu^{\sum_{i=1}^{l} n_{i} k(r-1) / 2} \cdot \sum_{\lambda \vdash r n} c_{\lambda^{1} \ldots \lambda^{l}}^{\lambda} \cdot t^{k \sum_{i=1}^{l} \kappa_{\lambda^{i}} / 2-k \kappa_{\lambda} / 2 r} \cdot s_{\lambda}^{*}(t, \nu) \\
& =t^{k r \sum_{i=1}^{l} \kappa_{\lambda^{i}} / 2} \cdot \nu^{k(r-1) n / 2} \cdot \sum_{\lambda \vdash r n} c_{\lambda^{1} \ldots \lambda^{l}}^{\lambda} \cdot t^{-k \kappa_{\lambda} / 2 r} \cdot s_{\lambda}^{*}(t, \nu) .
\end{aligned}
$$

The functions $s_{\lambda}^{*}(t, \nu)$ and the coefficients $c_{\lambda^{1} \ldots \lambda^{l}}^{\lambda}$ can be computed by using the Frobenius formula as follows. Let $\chi^{\lambda}$ and $C_{\mu}$ denote the character and conjugacy class of the symmetric group $\Sigma_{n}$ indexed by $\lambda, \mu \vdash n$. The Frobenius formula says that the Newton polynomial

$$
p^{\mu}\left(x_{1}, x_{2}, \ldots\right)=\prod_{i=1}^{\ell(\mu)} \sum_{j \geq 1} x_{j}^{\mu_{i}}
$$

is expressed in terms of Schur polynomials as

$$
p^{\mu}\left(x_{1}, x_{2}, \ldots\right)=\sum_{\lambda \vdash|\mu|} \chi^{\lambda}\left(C_{\mu}\right) \cdot s_{\lambda}\left(x_{1}, x_{2}, \ldots\right) .
$$

Its inverse for $\lambda \vdash n$ is

$$
s_{\lambda}\left(x_{1}, x_{2}, \ldots\right)=\sum_{\mu \vdash n} \frac{\left|C_{\mu}\right|}{n !} \chi^{\lambda}\left(C_{\mu}\right) \cdot p^{\mu}\left(x_{1}, x_{2}, \ldots\right) .
$$

Therefore, for the partition $\lambda \vdash n$ we have

$$
s_{\lambda}^{*}(t, \nu)=\sum_{\mu \vdash n} \frac{\left|C_{\mu}\right|}{n !} \chi^{\lambda}\left(C_{\mu}\right) \prod_{i=1}^{\ell(\mu)} \frac{\nu^{\mu_{i} / 2}-\nu^{-\mu_{i} / 2}}{t^{\mu_{i} / 2}-t^{-\mu_{i} / 2}} .
$$

Moreover, it is clear that

$$
p^{\mu^{1}}\left(x_{1}, x_{2}, \ldots\right) \cdot p^{\mu^{2}}\left(x_{1}, x_{2}, \ldots\right)=p^{\mu^{1}+\mu^{2}}\left(x_{1}, x_{2}, \ldots\right)
$$

and

$$
p^{\mu}\left(x_{1}^{r}, x_{2}^{r}, \ldots\right)=p^{\mu_{(r)}}\left(x_{1}, x_{2}, \ldots\right),
$$

where $\mu^{1}+\mu^{2}$ is the partition in which the number of each positive integer is the sum of those in $\mu^{1}, \mu^{2}$ and $\mu_{(r)}$ means the partition $\left(r \mu_{1}, r \mu_{2}, \ldots\right)$. Hence, for partitions $\lambda^{i} \vdash n_{i}, i=1, \ldots, l$ and $\lambda \vdash r\left(n_{1}+\cdots+n_{l}\right)$,

$$
c_{\lambda^{1} \ldots \lambda^{l}}^{\lambda}=\sum_{\mu^{1} \vdash n_{1}} \frac{\left|C_{\mu^{1}}\right|}{n_{1} !} \chi^{\lambda^{1}}\left(C_{\mu^{1}}\right) \cdots \sum_{\mu^{l} \vdash n_{l}} \frac{\left|C_{\mu^{l}}\right|}{n_{l} !} \chi^{\lambda^{l}}\left(C_{\mu^{l}}\right) \cdot \chi^{\lambda}\left(C_{\left(\mu^{1}+\cdots+\mu^{l}\right)_{(r)}}\right) .
$$

We finish this section by offering the following sample calculations. 
Example 5.5. Torus knot $T(2, k), k \not \equiv 0(\bmod 2)$.

$$
\begin{aligned}
W_{(1)}(t, \nu)= & \nu^{k / 2}\left(t^{-k / 2} s_{(2)}^{*}(t, \nu)-t^{k / 2} s_{(1,1)}^{*}(t, \nu)\right), \\
W_{(2)}(t, \nu)= & \nu^{k}\left(t^{-k} s_{(4)}^{*}(t, \nu)-t^{k} s_{(3,1)}^{*}(t, \nu)+t^{2 k} s_{(2,2)}^{*}(t, \nu)\right), \\
W_{(1,1)}(t, \nu) & =\nu^{k}\left(t^{-2 k} s_{(2,2)}^{*}(t, \nu)-t^{-k} s_{(2,1,1)}^{*}(t, \nu)+t^{k} s_{(1,1,1,1)}^{*}(t, \nu)\right), \\
W_{(3)}(t, \nu)= & \nu^{3 k / 2}\left(t^{-3 k / 2} s_{(6)}^{*}(t, \nu)-t^{3 k / 2} s_{(5,1)}^{*}(t, \nu)\right. \\
& \left.+t^{7 k / 2} s_{(4,2)}^{*}(t, \nu)-t^{9 k / 2} s_{(3,3)}^{*}(t, \nu)\right), \\
W_{(2,1)}(t, \nu) & =\nu^{3 k / 2}\left(t^{-5 k / 2} s_{(4,2)}^{*}(t, \nu)-t^{-3 k / 2} s_{(4,1,1)}^{*}(t, \nu)-t^{-3 k / 2} s_{(3,3)}^{*}(t, \nu)\right. \\
& \left.+t^{3 k / 2} s_{(2,2,2)}^{*}(t, \nu)+t^{3 k / 2} s_{(3,1,1,1)}^{*}(t, \nu)-t^{5 k / 2} s_{(2,2,1,1)}^{*}(t, \nu)\right), \\
W_{(1,1,1)}(t, \nu) & =\nu^{3 k / 2}\left(t^{-9 k / 2} s_{(2,2,2)}^{*}(t, \nu)-t^{-7 k / 2} s_{(2,2,1,1)}^{*}(t, \nu)\right. \\
& \left.+t^{-3 k / 2} s_{(2,1,1,1,1)}^{*}(t, \nu)-t^{3 k / 2} s_{(1,1,1,1,1,1)}^{*}(t, \nu)\right) .
\end{aligned}
$$

In particular,

$$
W_{(1)}(t, \nu)=\frac{\nu^{1 / 2}-\nu^{-1 / 2}}{t^{1 / 2}-t^{-1 / 2}}\left(\frac{t^{\frac{k+1}{2}}-t^{-\frac{k+1}{2}}}{t-t^{-1}} \nu^{\frac{k-1}{2}}-\frac{t^{\frac{k-1}{2}}-t^{-\frac{k-1}{2}}}{t-t^{-1}} \nu^{\frac{k+1}{2}}\right) .
$$

Example 5.6. Torus $k n o t ~ T(3, k), k \neq \equiv 0(\bmod 3)$.

$$
\begin{aligned}
W_{(1)}(t, \nu)= & \nu^{k}\left(t^{-k} s_{(3)}^{*}(t, \nu)-s_{(2,1)}^{*}(t, \nu)+t^{k} s_{(1,1,1)}^{*}(t, \nu)\right), \\
W_{(2)}(t, \nu)= & \nu^{2 k}\left(t^{-2 k} s_{(6)}^{*}(t, \nu)-s_{(5,1)}^{*}(t, \nu)+t^{2 k} s_{(4,1,1)}^{*}(t, \nu)\right. \\
& \left.+t^{2 k} s_{(3,3)}^{*}(t, \nu)-t^{3 k} s_{(3,2,1)}^{*}(t, \nu)+t^{4 k} s_{(2,2,2)}^{*}(t, \nu)\right), \\
W_{(1,1)}(t, \nu) & =\nu^{2 k}\left(t^{-4 k} s_{(3,3)}^{*}(t, \nu)-t^{-3 k} s_{(3,2,1)}^{*}(t, \nu)+t^{-2 k} s_{(3,1,1,1)}^{*}(t, \nu)\right. \\
& \left.+t^{-2 k} s_{(2,2,2)}^{*}(t, \nu)-s_{(2,1,1,1,1)}^{*}(t, \nu)+t^{2 k} s_{(1,1,1,1,1,1)}^{*}(t, \nu)\right) .
\end{aligned}
$$

Example 5.7. Torus link $T(2,2 k)$.

$$
\begin{aligned}
& W_{(1),(1)}(t, \nu)=t^{-k} s_{(2)}^{*}(t, \nu)+t^{k} s_{(1,1)}^{*}(t, \nu), \\
& W_{(2),(1)}(t, \nu)=t^{-2 k} s_{(3)}^{*}(t, \nu)+t^{k} s_{(2,1)}^{*}(t, \nu), \\
& W_{(1,1),(1)}(t, \nu)=t^{-k} s_{(2,1)}^{*}(t, \nu)+t^{2 k} s_{(1,1,1)}^{*}(t, \nu), \\
& W_{(2),(2)}(t, \nu)=t^{-4 k} s_{(4)}^{*}(t, \nu)+s_{(3,1)}^{*}(t, \nu)+t^{2 k} s_{(2,2)}^{*}(t, \nu), \\
& W_{(2),(1,1)}(t, \nu)=t^{-2 k} s_{(3,1)}^{*}(t, \nu)+t^{2 k} s_{(2,1,1)}^{*}(t, \nu), \\
& W_{(1,1),(1,1)}(t, \nu)=t^{-2 k} s_{(2,2)}^{*}(t, \nu)+s_{(2,1,1)}^{*}(t, \nu)+t^{4 k} s_{(1,1,1,1)}^{*}(t, \nu) .
\end{aligned}
$$

In particular,

$$
W_{(1),(1)}(t, \nu)=\frac{\nu^{1 / 2}-\nu^{-1 / 2}}{t^{1 / 2}-t^{-1 / 2}}\left(\frac{t^{\frac{2 k-1}{2}}+t^{-\frac{2 k-1}{2}}}{t-t^{-1}} \nu^{\frac{1}{2}}-\frac{t^{\frac{2 k+1}{2}}+t^{-\frac{2 k+1}{2}}}{t-t^{-1}} \nu^{-\frac{1}{2}}\right) .
$$


Example 5.8. Torus link $T(3,3 k)$.

$$
\begin{aligned}
& W_{(1),(1),(1)}(t, \nu)=t^{-3 k} s_{(3)}^{*}(t, \nu)+2 s_{(2,1)}^{*}(t, \nu)+t^{3 k} s_{(1,1,1)}^{*}(t, \nu), \\
& W_{(2),(1),(1)}(t, \nu)=t^{-5 k} s_{(4)}^{*}(t, \nu)+2 t^{-k} s_{(3,1)}^{*}(t, \nu)+t^{k} s_{(2,2)}^{*}(t, \nu)+t^{3 k} s_{(2,1,1)}^{*}(t, \nu), \\
& W_{(1,1),(1),(1)}(t, \nu)=t^{-3 k} s_{(3,1)}^{*}(t, \nu)+t^{-k} s_{(2,2)}^{*}(t, \nu) \\
& \quad+2 t^{k} s_{(2,1,1)}^{*}(t, \nu)+t^{5 k} s_{(1,1,1,1)}^{*}(t, \nu) .
\end{aligned}
$$

\section{On the Labastida-Mariño-VAfa CONJeCture}

As before, we have an oriented link $\mathcal{L}$ with $l$ components. Define the generating function

$$
Z\left(\mathbf{x}_{1}, \ldots, \mathbf{x}_{l}\right)=\sum_{\lambda^{1}, \ldots, \lambda^{l}} W_{\lambda^{1}, \ldots, \lambda^{l}}(t, \nu) \cdot s_{\lambda^{l}}\left(\mathbf{x}_{1}\right) \cdots s_{\lambda^{l}}\left(\mathbf{x}_{l}\right),
$$

where each $\mathbf{x}_{i}=\left\{x_{i, 1}, x_{i, 2}, \ldots\right\}$ is a set of indeterminate variables and $\lambda^{i}$ runs over all partitions including the empty one (the unique partition of zero). When all $\lambda^{i}$ are empty, the summand gives rise to the leading term 1.

One can expand $\log Z\left(\mathbf{x}_{1}, \ldots, \mathbf{x}_{l}\right)$ as

$$
\log Z\left(\mathbf{x}_{1}, \ldots, \mathbf{x}_{l}\right)=\sum_{d=1}^{\infty} \sum_{\lambda^{1}, \ldots, \lambda^{l}} \frac{1}{d} f_{\lambda^{1}, \ldots, \lambda^{l}}\left(t^{d}, \nu^{d}\right) \cdot s_{\lambda^{1}}\left(\mathbf{x}_{1}^{d}\right) \cdots s_{\lambda^{l}}\left(\mathbf{x}_{l}^{d}\right),
$$

where $\mathbf{x}_{i}^{d}=\left\{x_{i, 1}^{d}, x_{i, 2}^{d}, \ldots\right\}$. See [1] for an explanation why such an expansion exists by using the so-called plethystic exponential. The functions $f_{\lambda^{1}, \ldots, \lambda^{l}}(t, \nu)$ are referred to as the reformulated colored HOMFLY polynomial. The LabastidaMariño-Vafa conjecture says that these functions have the following highly nontrivial structure.

Write for $\lambda, \mu \vdash n$,

$$
M_{\lambda \mu}(t)=\sum_{\tau \vdash n} \frac{\left|C_{\tau}\right|}{n !} \chi^{\lambda}\left(C_{\tau}\right) \chi^{\mu}\left(C_{\tau}\right) \cdot \frac{\prod_{j=1}^{\ell(\tau)}\left(t^{-\tau_{j} / 2}-t^{\tau_{j} / 2}\right)}{t^{-1 / 2}-t^{1 / 2}} .
$$

Conjecture 6.1 (Labastida-Mariño-Vafa [11, [12]). For partitions $\lambda^{1}, \ldots, \lambda^{l}$,

$$
\begin{aligned}
& f_{\lambda^{1}, \ldots, \lambda^{l}}(t, \nu)=\sum_{\mu^{1} \vdash\left|\lambda^{1}\right|, \ldots, \mu^{l} \vdash\left|\lambda^{l}\right|} \hat{f}_{\mu^{1}, \ldots, \mu^{l}}(t, \nu) \cdot M_{\lambda^{1} \mu^{1}}(t) \cdots M_{\lambda^{l} \mu^{l}}(t), \\
& \hat{f}_{\mu^{1}, \ldots, \mu^{l}}(t, \nu)=\sum_{g \geq 0} \sum_{Q} N_{\mu^{1}, \ldots, \mu^{l}, g, Q} \cdot\left(t^{1 / 2}-t^{-1 / 2}\right)^{2 g+l-2} \cdot \nu^{Q},
\end{aligned}
$$

where $N_{\mu^{1}, \ldots, \mu^{l}, g, Q}$ are integers and $Q$ are either all integers or all semi-integers.

Moreover, the integers $N_{\mu^{1}, \ldots, \mu^{l}, g, Q}$ are interpreted as quantities involved in the enumerative geometry of the resolved conifold. See [5], [16] for example.

Until now, besides the trivial links, the conjecture was verified only for some simplest knots and links with small partitions. A proof of the Labastida-MariñoVafa conjecture seems to appeal to a deep knowledge of mathematics and string theory. Using the formula in the previous section, we can verify this conjecture for several infinite families of torus links with small partitions. Our calculation also suggests a new structure of the reformulated colored HOMFLY polynomial of torus links. Let us make this more precise first. 
Define symmetric functions for $\lambda, \mu \vdash n$,

$$
S_{\lambda, \mu}(\mathbf{x})=\sum_{\tau \vdash n} \frac{\left|C_{\tau}\right|}{n !} \chi^{\lambda}\left(C_{\tau}\right) \chi^{\mu}\left(C_{\tau}\right) \cdot p^{\tau}(\mathbf{x})
$$

and

$$
\begin{aligned}
s_{\mu ; q}(\mathbf{x}) & =\sum_{\lambda \vdash n}\left(q-q^{-1}\right) M_{\lambda \mu}\left(q^{-2}\right) \cdot s_{\lambda}(\mathbf{x}) \\
& =\sum_{\tau \vdash n} \frac{\left|C_{\tau}\right|}{n !} \chi^{\mu}\left(C_{\tau}\right) \cdot \prod_{j=1}^{\ell(\tau)}\left(q^{\tau_{j}}-q^{-\tau_{j}}\right) \cdot p^{\tau}(\mathbf{x}) .
\end{aligned}
$$

We have the following conjecture for torus links.

Conjecture 6.2. For torus link $T(r l, k l)$ with $r, k$ relatively prime and $n=n_{1}+$ $\cdots+n_{l}$,

$$
\begin{aligned}
& \left.\sum_{\lambda^{1} \vdash n_{1}, \ldots, \lambda^{l} \vdash n_{l}} f_{\lambda^{1}, \ldots, \lambda^{l}}(t, \nu) \cdot s_{\lambda^{1}}\left(\mathbf{x}_{1}\right) \cdots s_{\lambda^{l}}\left(\mathbf{x}_{l}\right)\right|_{t^{1 / 2}=q^{-1}, \nu^{1 / 2}=q^{-N}} \\
= & \sum_{\lambda^{1} \vdash n_{1}, \ldots, \lambda^{l} \vdash n_{l}}\left(q^{k}-q^{-k}\right)^{-2} \cdot q^{-k(r-1) n N} \cdot \sum_{\lambda \vdash r n} g_{\lambda^{1}, \ldots, \lambda^{l}}^{\lambda}\left(q^{2 k}\right) \\
\cdot & s_{\lambda ; q^{k}}\left(q^{N-1}, q^{N-3}, \ldots, q^{-(N-1)}\right) \cdot s_{\lambda^{1} ; q^{k}}\left(\mathbf{x}_{1}\right) \cdots s_{\lambda^{l} ; q^{k}}\left(\mathbf{x}_{l}\right),
\end{aligned}
$$

where $g_{\lambda^{1}, \ldots, \lambda^{l}}^{\lambda}(t) \in \mathbb{Z}\left[t^{ \pm 1}\right]$ is invariant under $t \rightarrow t^{-1}$.

The following examples verify Conjecture 6.2 .

Example 6.3. Torus knot $T(2, k), k \neq \equiv 0(\bmod 2)$. The following are nonvanishing $g_{\lambda^{\prime}}^{\lambda}(t)^{\prime}$ 's for $\left|\lambda^{\prime}\right| \leq 4$ :

$$
\begin{aligned}
& g_{(1)}^{(2)}(t)=1 \\
& g_{(1,1)}^{(2,2)}(t)=1 \\
& g_{(1,1,1)}^{(2,2,2)}(t)=t+t^{-1}, \\
& g_{(2,1)}^{(2,2,2)}(t)=g_{(1,1,1)}^{(3,2,1)}(t)=1 \\
& g_{(1,1,1,1)}^{(2,2,2,2)}(t)=t^{3}+2 t+1+2 t^{-1}+t^{-3}, \\
& g_{(2,1,1)}^{(2,2,2,2)}(t)=g_{(1,1,1,1)}^{(3,2,2,1)}(t)=t^{2}+t+2+t^{-1}+t^{-2}, \\
& g_{(2,1,1)}^{(3,2,2,1)}(t)=t+1+t^{-1}, \\
& g_{(2,2)}^{(2,2,2,2)}(t)=g_{(1,1,1,1)}^{(4,2,2)}(t)=g_{(1,1,1,1)}^{(3,3,1,1)}(t)=t+t^{-1}, \\
& g_{(3,1)}^{(2,2,2,2)}(t)=g_{(2,2)}^{(3,2,2,1)}(t)=g_{(2,1,1)}^{(4,2,2)}(t)=g_{(2,1,1)}^{(3,3,1,1)}(t)=1, \\
& g_{(1,1,1,1)}^{(4,3,1)}(t)=g_{(1,1,1,1)}^{(4,2,1,1)}(t)=g_{(1,1,1,1)}^{(3,3,2)}(t)=1 .
\end{aligned}
$$


Example 6.4. Torus knot $T(3, k), k \neq \equiv 0(\bmod 3)$. The following are nonvanishing $g_{\lambda^{\prime}}^{\lambda}(t)$ 's for $\left|\lambda^{\prime}\right| \leq 3$ :

$$
\begin{aligned}
& g_{(1)}^{(3)}(t)=1 \\
& g_{(1,1)}^{(3,3)}(t)=t+t^{-1} \\
& g_{(2)}^{(3,3)}(t)=g_{(1,1)}^{(4,2)}(t)=1 \\
& g_{(1,1,1)}^{(3,3,3)}(t)=t^{4}+2 t^{2}+2 t+2+2 t^{-1}+2 t^{-2}+t^{-4} \\
& g_{(2,1)}^{(3,3,3)}(t)=g_{(1,1,1)}^{(4,3,2)}(t)=t^{3}+t^{2}+2 t+3+2 t^{-1}+t^{-2}+t^{-3} \\
& g_{(2,1)}^{(4,3,2)}(t)=t^{2}+2 t+2+2 t^{-1}+t^{-2} \\
& g_{(1,1,1)}^{(5,3,1)}(t)=t^{2}+t+2+t^{-1}+t^{-2} \\
& g_{(2,1)}^{(5,3,1)}(t)=t+1+t^{-1}, \\
& g_{(3)}^{(3,3,3)}(t)=g_{(1,1,1)}^{(6,3)}(t)=g_{(1,1,1)}^{(5,2,2)}(t)=g_{(1,1,1)}^{(4,4,1)}(t)=t+t^{-1}, \\
& g_{(3)}^{(4,3,2)}(t)=g_{(2,1)}^{(6,3)}(t)=g_{(2,1)}^{(5,2,2)}(t)=g_{(2,1)}^{(4,4,1)}(t)=g_{(1,1,1)}^{(6,2,1)}(t)=g_{(1,1,1)}^{(5,4)}(t)=1 .
\end{aligned}
$$

Example 6.5. Torus link $T(2,2 k)$. The following are nonvanishing $g_{\lambda^{1}, \lambda^{2}}^{\lambda}(t)$ 's for $\left|\lambda^{1}\right|+\left|\lambda^{2}\right| \leq 5$, up to symmetry $g_{\lambda^{1}, \lambda^{2}}^{\lambda}(t)=g_{\lambda^{2}, \lambda^{1}}^{\lambda}(t)$ :

$$
\begin{aligned}
& g_{(0),(1)}^{(1)}(t)=g_{(1),(1)}^{(2)}(t)=g_{(2),(1)}^{(3)}(t)=g_{(3),(1)}^{(4)}(t)=g_{(4),(1)}^{(5)}(t)=1, \\
& g_{(2),(2)}^{(4)}(t)=t+1+t^{-1} \\
& g_{(2),(1,1)}^{(4)}(t)=g_{(2),(2)}^{(3,1)}(t)=1 \\
& g_{(3),(2)}^{(5)}(t)=t^{2}+t+3+t^{-1}+t^{-2} \\
& g_{(3),(2)}^{(4,1)}(t)=g_{(3),(1,1)}^{(5)}(t)=g_{(2,1),(2)}^{(5)}(t)=t+1+t^{-1}, \\
& g_{(3),(2)}^{(3,2)}(t)=g_{(3),(1,1)}^{(4,1)}(t)=g_{(2,1),(2)}^{(4,1)}(t)=g_{(2,1),(1,1)}^{(5)}(t)=1 .
\end{aligned}
$$

One notices that (6.4) is indeed equivalent to

$$
\begin{aligned}
& \sum_{\lambda^{1} \vdash n_{1}, \ldots, \lambda^{l} \vdash n_{l}} f_{\lambda^{1}, \ldots, \lambda^{l}}(t, \nu) \cdot s_{\lambda^{1}}\left(\mathbf{x}_{1}\right) \cdots s_{\lambda^{l}}\left(\mathbf{x}_{l}\right) \\
= & \sum_{\mu^{1} \vdash n_{1}, \ldots, \mu^{l} \vdash n_{l}}\left(t^{-1 / 2}-t^{1 / 2}\right)^{-l} \cdot \hat{f}_{\mu^{1}, \ldots, \mu^{l}}(t, \nu) \cdot s_{\mu^{1} ; t^{-1 / 2}}\left(\mathbf{x}_{1}\right) \cdots s_{\mu^{l} ; t^{-1 / 2}}\left(\mathbf{x}_{l}\right) .
\end{aligned}
$$

Therefore, it follows from the identities

$$
\begin{aligned}
& s_{\lambda ; q^{k}}\left(q^{N-1}, q^{N-3}, \ldots, q^{-(N-1)}\right)=s_{\lambda ; q^{N}}\left(q^{k-1}, q^{k-3}, \ldots, q^{-(k-1)}\right), \\
& s_{\lambda ; q^{k}}(\mathbf{x})=\sum_{\mu \vdash|\lambda|} S_{\lambda, \mu}\left(q^{k-1}, q^{k-3}, \ldots, q^{-(k-1)}\right) \cdot s_{\mu ; q}(\mathbf{x})
\end{aligned}
$$


that (6.8) implies

$$
\begin{gathered}
\hat{f}_{\mu^{1}, \ldots, \mu^{l}}(t, \nu)=\left(t^{-1 / 2}-t^{1 / 2}\right)^{l-2} \cdot\left(\frac{t^{k / 2}-t^{-k / 2}}{t^{1 / 2}-t^{-1 / 2}}\right)^{-2} \cdot \nu^{k(r-1) n / 2} \\
\cdot \sum_{\lambda^{1} \vdash n_{1}, \ldots, \lambda^{l} \vdash n_{l}} \prod_{i=1}^{l} S_{\lambda^{i}, \mu^{i}}\left(t^{(k-1) / 2}, t^{(k-3) / 2}, \ldots, t^{-(k-1) / 2}\right) \\
\cdot \sum_{\lambda \vdash r n} g_{\lambda^{1}, \ldots, \lambda^{l}}^{\lambda}\left(t^{k}\right) \cdot s_{\lambda ; \nu^{-1 / 2}}\left(t^{(k-1) / 2}, t^{(k-3) / 2}, \ldots, t^{-(k-1) / 2}\right)
\end{gathered}
$$

for partitions $\mu^{i} \vdash n_{i}, i=1, \ldots, l$ and $n=n_{1}+\cdots+n_{l}$.

Let us take $T(2, k), k \neq \equiv 0(\bmod 2)$, as an example to illustrate our verification of the Labastida-Mariño-Vafa conjecture for torus links. In this case, we have

$$
\begin{aligned}
& \hat{f}_{(2)}(t, \nu)=-\nu^{k}\left(\nu^{\frac{1}{2}}-\nu^{-\frac{1}{2}}\right)^{2}\left(\nu+\nu^{-1}-t-t^{-1}\right) \\
& \cdot \frac{\left(t^{\frac{k+1}{2}}-t^{-\frac{k+1}{2}}\right)\left(t^{\frac{k}{2}}-t^{-\frac{k}{2}}\right)\left(t^{\frac{k-1}{2}}-t^{-\frac{k-1}{2}}\right)^{2}}{\left(t^{\frac{3}{2}}-t^{-\frac{3}{2}}\right)\left(t-t^{-1}\right)^{3}\left(t^{\frac{1}{2}}-t^{-\frac{1}{2}}\right)}, \\
& \hat{f}_{(1,1)}(t, \nu)=-\nu^{k}\left(\nu^{\frac{1}{2}}-\nu^{-\frac{1}{2}}\right)^{2}\left(\nu+\nu^{-1}-t-t^{-1}\right) \\
& \quad \cdot \frac{\left(t^{\frac{k+1}{2}}-t^{-\frac{k+1}{2}}\right)^{2}\left(t^{\frac{k}{2}}-t^{-\frac{k}{2}}\right)\left(t^{\frac{k-1}{2}}-t^{-\frac{k-1}{2}}\right)}{\left(t^{\frac{3}{2}}-t^{-\frac{3}{2}}\right)\left(t-t^{-1}\right)^{3}\left(t^{\frac{1}{2}}-t^{-\frac{1}{2}}\right)} .
\end{aligned}
$$

Since $k$ is odd, both functions agree with the Labastida-Mariño-Vafa conjecture. Following this method we can verify the Labastida-Mariño-Vafa conjecture for all the torus links in Examples 6.3, 6.4 and 6.5.

\section{ACKNOWLEDGMENTS}

The authors would like to thank Professors Kefeng Liu and Jian Zhou for their interest in this work.

\section{REFERENCES}

1. A. K. Aiston and H. R. Morton, Idempotents of Hecke algebras of type A, J. Knot Theory Ramif. 7 (1998), 463-487. MR.1633027 (99h:57002)

2. J. S. Birman and H. Wenzl, Braids, link polynomials and a new algebra, Trans. Amer. Math. Soc. 313 (1989), 249-273. MR992598 (90g:57004)

3. R. Dipper and G. James, Representations of Hecke algebras of general linear groups, Proc. London Math. Soc. 52 (1986), 20-52. MR812444 (88b:20065)

4. R. Dipper and G. James, Blocks and idempotents of Hecke algebras of general linear groups, Proc. London Math. Soc. 54 (1987), 57-82. MR872250 (88m:20084)

5. R. Gopakumar and C. Vafa, On the gauge theory/geometry correspondence, Adv. Theor. Math. Phys. 3 (1999), 1415-1443. MR 1796682 (2001k:81272)

6. A. Gyoja, A q-analogue of Young symmetrizer, Osaka J. Math. 23 (1986), 841-852. MR $873212(88 \mathrm{e}: 20012)$

7. V. Jones, Hecke algebra representations of braid groups and link polynomials, Ann. Math. 126 (1987), 335-388. MR908150 (89c:46092)

8. C. Kassel, Quantum Groups, Graduate Texts in Mathematics 155, Springer-Verlag, 1995. MR 1321145 (96e:17041)

9. A. Klimyk and K. Schmüdgen, Quantum Groups and Their Representations, Springer-Verlag, 1997. MR:1492989 (99f:17017)

10. J. M. F. Labastida and M. Mariño, Polynomial invariants for torus knots and topological strings, Comm. Math. Phys. 217 (2001), 423-449. MR.1821231 (2003b:57016)

11. J. M. F. Labastida and M. Mariño, A new point of view in the theory of knot and link invariants, J. Knot Theory Ramif. 11 (2002), 173-197. MR.1895369 (2003h:57016) 
12. J. M. F. Labastida, M. Mariño and C. Vafa, Knots, links and branes at large N, J. High Energy Phys. 2000, no. 11, Paper 7, 42 pp. MR.1806596 (2003b:57015)

13. R. Leduc and A. Ram, A ribbon Hopf algebra approach to the irreducible representations of centralizer algebras: The Brauer, Birman-Wenzl, and type A Iwahori-Hecke algebras, Adv. Math. 125 (1997), 1-94. MR 1427801 (98c:20015)

14. H. Morton and S. Lukac, The Homfly polynomial of the decorated Hopf link, J. Knot Theory Ramif. 12 (2003), 395-416. MR1983094 (2004d:57012)

15. G. Murphy, On the representation theory of the symmetric groups and associated Hecke algebras, J. Algebra 152 (1992), 492-513. MR.1194316 (94c:17031)

16. H. Ooguri and C. Vafa, Knot invariants and topological strings, Nucl. Phys. B 577 (2000), 419-438. MR1765411 (2001i:81254)

17. N. Yu. Reshetikhin and V. G. Turaev, Ribbon graphs and their invariants derived from quantum groups, Comm. Math. Phys. 127 (1990), 1-26. MR1036112 (91c:57016)

18. B. E. Sagan, The Symmetric Group: Representations, Combinatorial Algorithms, and Symmetric Functions, Wadsworth Inc., Belmont, California, 1991. MR.1093239 (93f:05102)

19. V. G. Turaev, The Yang-Baxter equation and invariants of links, Invent. Math. 92 (1988), 527-553. MR939474 (89e:57003)

20. H. Wenzl, Quantum groups and subfactors of type B, C, and D, Comm. Math. Phys. 133 (1990), 383-432. MR1090432 (92k:17032)

21. E. Witten, Quantum field theory and the Jones polynomial, Comm. Math. Phys. 121 (1989), 351-399. MR990772 (90h:57009)

Department of Mathematics, University of California, Riverside, California 92521

Department of Mathematics, Zhongshan University, Guangzhou, Guangdong 510275, People's Republic of China

E-mail address: zhenghao@mail.sysu.edu.cn 